\title{
Construction of a minimum energy path for the VT flash model by the string method coupled with the exponential time differencing scheme
}

\author{
Yuze Zhang ${ }^{1}$, Yiteng $\mathrm{Li}^{2}$, Lei Zhang ${ }^{1}$, Shuyu Sun ${ }^{2, *}$ \\ ${ }^{1}$ Beijing International Center for Mathematical Research, Peking University, Beijing \\ 100871, China \\ ${ }^{2}$ Physical Science and Engineering Division (PSE), King Abdullah University of \\ Science and Technology (KAUST), Thuwal 23955-6900, Saudi Arabia
}

\begin{abstract}
Flash calculation plays significant roles in various aspects of petroleum and chemical industries. Since Michelsen proposed his milestone studies in 1982, through several decades of development, the current research interests on flash calculation have been shifted from accuracy to efficiency, but the ultimate goal remains the same; that is accurate determination of equilibrium phase amounts and compositions at a given condition. On the other hand, finding the transition route and its related saddle point is often crucial to understand the whole energy landscape of flash models, which would provide new insights for designing numerical algorithms or optimizing existing ones. In this study, an efficient numerical approach is developed by coupling the string method with the exponential time differencing (ETD) scheme to investigate the minimum energy paths and first-order saddle points of VT flash models with PengRobinson equation of state. As a promising alternative to the conventional approach, VT flash calculates phase equilibria under a new variable specification of volume and temperature. The Rosenbrock-type ETD scheme is used to reduce the computational difficulty caused by the high stiffness of the model systems. The proposed ETD-String method successfully calculates the minimum energy paths of single-component and two-component VT flash models with strong stiffness. Numerical results also show good feasibility and accuracy in calculation of equilibrium phase amounts and compositions.
\end{abstract}

Key words: VT flash, Peng-Robinson equation of state, minimum energy path, string method, Rosenbrock-type exponential time differencing scheme

\footnotetext{
${ }^{*}$ Corresponding author. Email addresses: 1906391168@pku.edu.cn (Y.Z. Zhang), yiteng.li@kaust.edu.sa (Y.T. Li), zhangl@math.pku.edu.cn (L. Zhang), shuyu. sun@kaust. edu.sa (S.Y. Sun)
} 


\section{Introduction}

Accurate knowledge of phase equilibria is of vital importance in petroleum industry to determine the number of equilibrium phases and their amounts and compositions for complex reservoir fluids, which stimulates the development of equation-of-state-based flash calculation. One important application of flash calculation is modeling phase behaviors of hydrocarbon mixtures in compositional flow simulators, which are mainly used to model recovery processes sensitive to compositional changes, such as miscible flooding [1]. Moreover, it is often used as standalone calculation as well to analyze, design and optimize processes and facilities, such as reducing undesired species (e.g. $\mathrm{H}_{2} \mathrm{O}$ ) for conforming to product specifications, removing acid gas (e.g. $\mathrm{CO}_{2}$ and $\mathrm{H}_{2} \mathrm{~S}$ ) for protecting pipelines and equipment from corrosion [2-4], determining the amount of inhibitor (e.g. methanol and glycols) for avoiding gas hydrate formation [5- 8$]$, controlling asphaltene precipitation for enhancing flow assurance [9-11], etc.

The most commonly-used flash model of a $M$ components system is formulated under the specified pressure $(P)$, temperature $(T)$ and chemical composition $\left(N=\left[N_{1}, \ldots, N_{M}\right]\right)$, which is known as PT flash calculation in petroleum industry. Usually, the Wilson correlation is used to initialize flash calculation but this approximation does not guarantee convergence to the equilibrium solution. To overcome this issue, stability test is applied which gives a better initial approximation but meanwhile requires additional computational cost. The milestone studies of Michelsen in stability testing [12] and phase splitting calculation [13] laid a foundation for the development of PT flash calculation using a single consistent equation of state (EOS), such as the Soave-Redlich-Kwong (SRK) EOS [14] and the Peng-Robinson (PR) EOS [15].

Recently, the computation of phase equilibria under the given volume $(V)$, temperature $(T)$ and mole composition $(N)$, also known as VT flash calculation, has become a promising alternative to the conventional PT flash model. In addition to its well-posed formulation, VT flash exhibits a unique pressure-volume relation so that the root selection procedure in PT flash framework can be avoided [16]. Another important advantage of VT flash formulation is modeling phase behaviors of unconventional reservoir fluids with nonnegligible capillary effect [17-19], given that the commonly-used interfacial tension models are explicit functions of molar density rather than pressure. Moreover, it is straightforward to use VT flash to model complex fluid mixtures that contain associating fluid, like $\mathrm{H}_{2} \mathrm{O}$, since the association contribution is originally defined based on Helmholtz free energy. During the past few years, numerous efforts have been made to expand the applications of VT flash calculation not only in various phase equilibria 
problems [20-22] but also in compositional flow simulation [23].

The aforementioned flash calculations mainly focus on the determination of phase amounts and compositions at the equilibrium state where either the Gibbs free energy or Helmholtz free energy is minimized at the end of PT or VT flash calculation. In principle, the equilibrium state calculated by the classical flash framework is usually a local minimum of the energy landscape, but there is no guarantee it is the one with the lowest energy. Although a variety of global minimization methods were developed to solve flash problems [24-27], their numerical efficiency heavily restricts their applications. To study the whole energy landscape of flash models, an attractive approach is to find transition routes between different local minima. These transition routes are called as minimum energy paths (MEPs). In particular, we can obtain the first-order saddle points' information of the energy landscape from the MEPs, which are the dynamical bottlenecks, also known as energy barriers, in the transition state theory. Furthermore, the knowledge of high-order saddle points is also helpful in finding multiple local minima [29-31], as well as the relative minimum point of energy stability. Thus, a comprehensive understanding of saddle points' information would help to locate the global minimum, which further demonstrates that the equilibrium point calculated by the classical flash framework is the one with the lowest energy. Such knowledge could provide valuable information to design and improve numerical schemes as well as algorithms for flash calculations.

In this study, we investigate the computation of the MEP and the first-order saddle points of VT flash models. There are several numerical schemes proposed to calculate the MEPs [28, 32, 36]. Among them, the string method [28, 35] can efficiently determine the energy transition pathways for complex systems with smooth energy landscape by evolving strings rather than points in configuration space. This approach consists of two main steps, the evolution of the string by some ordinary differential equation (ODE) solvers and the reparametrization of the string by interpolation. The accuracy of this approach significantly relies on ODE solvers. It is worth mentioning that equation-of-state-based VT flash models could have ill-conditioned Hessian matrices with very large condition numbers (For example, in the single-component example of this study, the two eigenvalues of the Hessian matrix differ by $10^{8}-10^{9}$ times). Moreover, explicit numerical approaches are required because the real scale of the problem is huge. Some common ODE solvers, like Euler and Runge-Kutta methods, require a very small time step for solving ill-conditioned problems. Instead, an exponential time differencing (ETD) scheme is used here to reduce the influence of the rigid term during numerical calculation.

The ETD scheme involves exact integration of the governing equations followed by an explicit approximation of a temporal integral for the nonlinear terms. It can provide 
satisfactory accuracy and stability even for problems with strong stiffness. This scheme was originally applied in the field of computational electrodynamics [37]. Then it was systematically studied in [38] and was developed for solving stiff systems by Cox and Matthews [39]. Also, in [39], high-order multi-step ETD schemes and Rounge-Kutta versions of ETD schemes were discussed. The linear stabilities of some ETD and modified ETD schemes were investigated by Du and Zhu [40, 41], and the corresponding ETD algorithms were then analyzed in [42-44]. For some problems with very strong stiff terms, a bad chosen linearization of ordinary ETD schemes may cause a severe step size restriction. To avoid these problems, Hochbruck and Ostermann proposed Rosenbrock-type ETD scheme [45, 46] to handle the stiff nonlinear term without loss of the original stability. Thus, in this study, a novel ETD-String method is proposed to compute the MEPs and the first-order saddle points of VT flash models.

The structure of this paper is organized as follows. In Section 2, the conventional VT flash model is briefly introduced. In Section 3, we establish the ETD-String method for calculating the MEPs of VT flash model. In Section 4, numerical examples are presented to demonstrate the performance of the proposed ETD schemes and construct the MEPs for single-component and two-component VT flash models. We make some remarks and conclusions in Section 5.

\section{VT flash calculation}

Let us consider a reservoir fluid mixture of $M$ components occupies volume $V$ at temperature $T$. If the investigated mixture is unstable and splits into at most two phases, the total Helmholtz free energy of a two-phase system is given by

$$
F_{\text {total }}=F_{1}+F_{2}=F\left(N_{1}, V_{1}, T\right)+F\left(N_{2}, V_{2}, T\right),
$$

where $N_{\alpha}=\left[N_{1, \alpha}, \ldots, N_{M, \alpha}\right]^{T}$ and $V_{\alpha}$ denote the mole number vector and volume of phase $\alpha(\alpha=1,2)$. The expression of the Helmholtz free energy $F$ has the following form

$$
F\left(N_{\alpha}, V_{\alpha}\right)=R T \sum_{i=1}^{M} N_{i, \alpha}\left(\ln \frac{N_{i, \alpha}}{V_{\alpha}}-1\right)-N_{t, \alpha} R T \ln \left(\frac{V_{\alpha}-B_{\alpha}}{V_{\alpha}}\right)+\frac{A_{\alpha}}{2 \sqrt{2} B_{\alpha}} \ln \left(\frac{V_{\alpha}+(1-\sqrt{2}) B_{\alpha}}{V_{\alpha}+(1+\sqrt{2}) B_{\alpha}}\right),
$$

where $N_{t, \alpha}=\sum_{i} N_{i, \alpha}$ is the total mole number of phase $\alpha$. The definition of $A_{\alpha}$ and $B_{\alpha}$ can be found in Appendix A For brevity, we will drop $T$ in the following expressions since $T$ is held constant.

The second law of thermodynamics indicates the minimum principle of Helmholtz free energy under specified $N, V$ and $T$ [47]. Thus, the phase equilibrium problem can be 
formulated as the following constrained minimization problem if $N_{1}$ and $V_{1}$ are chosen as the primary unknown variables

$$
\min F\left(N_{1}, V_{1}\right)
$$

subject to

$$
\begin{array}{r}
0 \leq N_{i, 1} \leq N_{i}, \\
V_{1}-\sum_{i=1}^{M} b_{i} N_{i, 1}>0, \\
\left(V-V_{1}\right)-\sum_{i=1}^{M} b_{i}\left(N_{i}-N_{i, 1}\right)>0,
\end{array}
$$

In particular, 2.4a) results from the mole conservation constraint $\left(N_{i, 1}+N_{i, 2}=N_{i}, i=\right.$ $1, \ldots, M)$, namely neither $N_{i, 1}$ nor $N_{i, 2}$ is allowed to be negative. The constraints in 2.4b and (2.4c) ensures the calculation of $F$ are always meaningful for both phases, with $B_{\alpha}$ is defined as the summation of the product of $b_{i}$ and $N_{i, \alpha}$. Here $b_{i}$ is the co-volume parameter of component $i$ and it is calculated by the critical pressure $\left(P_{c}\right)$, critical temperature $\left(T_{c}\right)$, both of which are given parameters.

To efficiently solve bulk phase equilibria problems, one popular approach is making use of the local curvature of the energy function. The symmetry of Hessian matrices has been extensively used to design fast and robust numerical algorithms for VT flash calculations [16, 48,-51]. With a proper line search scheme, the constrained minimization problem, comprising (2.3) and 2.4a)-2.4c), can be reformulated as a equation-solving problem. To ensure a constant decline of Helmholtz free energy over iterations, the Hessian matrix is required to be positive definite as indicated in the aforementioned literature. Thus, for the sake of safety, the positive definiteness of the Hessian matrix is examined at each iteration, and a modified Cholesky factorization [52, 53] is performed if it is found not sufficiently positive definite. Clearly, such a process requires additional operations on the Hessian matrix. In contrast, the proposed numerical algorithm, which combines the ETD scheme and the string method, circumvents the modified Cholesky algorithm. In this study, the VT flash model is solved based on the following ODE system

$$
\begin{gathered}
\frac{\partial N_{i, 1}}{\partial t}=-\mu_{i}\left(N_{1}, V_{1}\right) V_{1}+\mu_{i}\left(N_{2}, V_{2}\right) V_{2}, \quad i=1, \ldots, M \\
\frac{\partial V_{1}}{\partial t}=\frac{1}{V_{1}}\left(\sum_{i=1}^{M} \mu_{i}\left(N_{1}, V_{1}\right) N_{i, 1}-F\left(N_{1}, V_{1}\right)\right)-\frac{1}{V_{2}}\left(\sum_{i=1}^{M} \mu_{i}\left(N_{2}, V_{2}\right) N_{i, 2}-F\left(N_{2}, V_{2}\right)\right),
\end{gathered}
$$


where $\mu_{i}$ is the chemical potential of component $i$ and its formulation is shown below

$$
\begin{aligned}
\mu_{i}\left(N_{\alpha}, V_{\alpha}\right)= & R T \ln \left(\frac{N_{i, \alpha}}{V_{\alpha}}\right)-R T \ln \left(\frac{V_{\alpha}-B_{\alpha}}{V_{\alpha}}\right)+\frac{N_{t, \alpha} R T b_{i}}{V_{\alpha}-B_{\alpha}}+ \\
& \frac{\left(2 \sum_{k} a_{i k} N_{k, \alpha}\right) B_{\alpha}-A_{\alpha} b_{i}}{2 \sqrt{2} B_{\alpha}^{2}} \ln \left(\frac{V_{\alpha}+(1-\sqrt{2}) B_{\alpha}}{V_{\alpha}+(1+\sqrt{2}) B_{\alpha}}\right)-\frac{A_{\alpha}}{B_{\alpha}}\left(\frac{b_{i}}{V_{\alpha}^{2}+2 B_{\alpha} V_{\alpha}-B_{\alpha}^{2}}\right) .
\end{aligned}
$$

It is worth mentioning (2.5) implicitly takes the constraints (2.4) into account to make sure the calculation of $\mu_{i}$ and $F$ confines to real numbers.

\section{The ETD-String method}

The main purpose of using the string method is to find the MEPs for barrier-crossing events. If the potential energy $F$ of a system has at least two local minima $m_{1}$ and $m_{2}$, a MEP is a curve $\gamma$ which connects these two local minima and tangents to $\nabla F$

$$
\nabla F(\gamma)-(\nabla F(\gamma), \hat{\gamma}) \hat{\gamma}=0
$$

where $\hat{\gamma}$ denotes the unit tangent of $\gamma$. Basically, finding the MEP by string is to evolve a curve connecting two local minima under some certain dynamics and the overall algorithm is an iterative application of two steps: (1) string evolution and (2) string reparametrization.

String evolution: For the first step, since $N_{i}$ and $V$ are fixed, (2.5) can be transformed into a system with respect to primary unknowns $\boldsymbol{u}=\left[N_{1,1}, \ldots, N_{M, 1}, V_{1}\right]$. As a result, $N$ discrete points $\boldsymbol{u}_{i}=\left[N_{i, 1,1}, \ldots, N_{i, M, 1}, V_{i, 1}\right]$ along the string can be evolved by VT flash dynamics

$$
\left(\boldsymbol{u}_{i}\right)_{t}=-\nabla F\left(\boldsymbol{u}_{i}\right), \quad i=1, \ldots, N .
$$

For VT flash problems with strong stiffness (3.2), conventional ODE solvers require a very small time step and sometimes have large errors. However, Rosenbrock-Euler ETD schemes could effectively reduce the numerical difficulty caused by the stiffness of the Hessian matrix. To apply the Rosenbrock-Euler ETD scheme, first a linear term is combined into the original system and the new system is written as

$$
\left(\boldsymbol{u}_{i}\right)_{t}=H\left(\boldsymbol{u}_{i, n}\right) \boldsymbol{u}_{i}+G\left(\boldsymbol{u}_{i}, \boldsymbol{u}_{i, n}\right), \quad i=1, \ldots, N
$$

where $H\left(\boldsymbol{u}_{i, n}\right)=H_{i, n}$ is the Hessian matrix of total energy, $\boldsymbol{u}_{i, n}=\left[N_{i, 1,1}, \ldots, N_{i, M, 1}, V_{i, 1}\right]_{n}$ is the variable set of system at $n_{t h}$ time step and

$$
G\left(\boldsymbol{u}_{i}, \boldsymbol{u}_{i, n}\right)=-\nabla F\left(\boldsymbol{u}_{i}\right)-H\left(\boldsymbol{u}_{i, n}\right) \boldsymbol{u}_{i}, \quad i=1, \ldots, N
$$


From (3.3), it gives

$$
\left(e^{-t H_{i, n}} \boldsymbol{u}\right)_{t}=e^{-t H_{i, n}} G\left(\boldsymbol{u}_{i}, \boldsymbol{u}_{i, n}\right), \quad i=1, \ldots, N .
$$

Then we integrate (3.4) on both sides from 0 to one time step $\tau$, which yields

$$
\begin{aligned}
& e^{-t_{n+1} H_{i, n}} \boldsymbol{u}_{i, n+1}-e^{-t_{n} H_{i, n}} \boldsymbol{u}_{i, n}=\int_{0}^{\tau} e^{-\left(t_{n}+s\right) H_{i, n}} G\left(\boldsymbol{u}_{i}\left(t_{n}+s\right), \boldsymbol{u}_{i, n}\right) d s, \\
& \boldsymbol{u}_{i, n+1}=e^{\tau H_{i, n}} \boldsymbol{u}_{i, n}+\int_{0}^{\tau} e^{(\tau-s) H_{i, n}} G\left(\boldsymbol{u}_{i}\left(t_{n}+s\right), \boldsymbol{u}_{i, n}\right) d s, \quad i=1, \ldots, N .
\end{aligned}
$$

Unlike ordinary ODE solvers, the Rosenbrock ETD scheme gives the exact relationship between $\boldsymbol{u}_{i, n+1}$ and $\boldsymbol{u}_{i, n}$. To some extent, the influence of Hessian matrix on the convergence performance is negligible and thus it allows us to avoid using the modified Cholesky algorithm, which is widely used to ensure a descend direction for local minimization methods. In addition, if different numerical methods are used to approximate $\boldsymbol{u}_{i}\left(t_{n}+s\right)$ in (3.5), we can get different ETD schemes for the system (2.5). For instance, by replacing $\boldsymbol{u}_{i}\left(t_{n}+s\right)$ with $\boldsymbol{u}_{i, n}$, the following Rosenbrock-Euler-ETD scheme is established

$$
\boldsymbol{u}_{i, n+1}=e^{\tau H_{i, n}} \boldsymbol{u}_{i, n}+H_{i, n}^{-1}\left(e^{\tau H_{i, n}}-I\right) G\left(\boldsymbol{u}_{i, n}, \boldsymbol{u}_{i, n}\right), \quad i=1, \ldots, N
$$

which can be rewritten into

$$
\boldsymbol{u}_{i, n+1}=e^{\tau H_{i, n}} \boldsymbol{u}_{i, n}+\tau \phi_{1}\left(\tau H_{i, n}\right) G\left(\boldsymbol{u}_{i, n}, \boldsymbol{u}_{i, n}\right), \quad i=1, \ldots, N
$$

where

$$
\phi_{1}(\alpha)=\left(e^{\alpha}-1\right) \alpha^{-1} .
$$

It can be seen from (3.7) that the inverse of the Hessian matrix is needed. To calculate it, for a diagonalizable matrix $\tau H_{i, n}$, a nonsingular matrix $P$ can be found such that $\tau H_{i, n}=$ $P^{-1} A P$, where $A=\operatorname{diag}\left(\lambda_{1} \ldots \lambda_{N}\right)$ and $\lambda_{i}$ is the $i_{\text {th }}$ eigenvalues of $\tau H_{i, n}$. According to [54],

$$
\phi_{1}\left(P^{-1} A P\right)=P^{-1} \phi_{1}(A) P=P^{-1}\left(\begin{array}{cccc}
\phi_{1}\left(\lambda_{1}\right) & 0 & \cdots & 0 \\
0 & \ddots & \ddots & \vdots \\
\vdots & \ddots & \ddots & 0 \\
0 & \cdots & 0 & \phi_{1}\left(\lambda_{N}\right)
\end{array}\right) P,
$$

If $H_{i, n}$ is singular, there exists zero eigenvalue $\lambda_{\text {zero }}$. Rather than calculating zero eigenvalue, it can be approximated by $\phi_{1}\left(\lambda_{\text {zero }}\right)=\lim _{\alpha \rightarrow 0} \phi_{1}(\alpha)$ instead.

String reparametrization: For the reparametrization procedure, it is worth pointing out that only the normal component of the curve's velocity affects the curve, while the tangential velocity only contributes to the movement of points along the curve. Thus, it is 
free to choose any parametrization and here a particular curve parametrization $\Gamma=\{\phi(\alpha)$ : $\alpha \in[0,1]\}$ is used. Whenever the curve evolves, points on the $\Gamma$ will change their positions following the dynamic (3.2). It is then necessary to interpolate these points and their values onto a same parametrization $\Gamma$. Here a parametrization is implemented based on equal arc length, which consists of the following two steps:

(1) Calculate the arc length $l_{i}$ based on the current point set $\left\{\boldsymbol{u}_{i}\right\}$ of the curve

$$
l_{i}=l_{i-1}+\left|\boldsymbol{u}_{i}-\boldsymbol{u}_{i-1}\right|, \quad i=1, \ldots, N,
$$

where $l_{0}=0$ and then the nonuniform mesh $\left\{m_{i}\right\}$ can be obtained by normalizing $\left\{l_{i}\right\}$

$$
m_{i}=l_{i} / l_{N} .
$$

(2) Estimate new $\boldsymbol{u}_{i}^{*}$ at the uniform mesh $\left\{m_{i}^{*}=i / N\right\}$ under given $\left\{m_{i}\right\}$ and $\left\{\boldsymbol{u}_{i}\right\}$ by interpolation.

To obtain a finer resolution around the saddle point, another parametrization process is carried out by energy-weight arc length. In contrast to the preceding method with equal arc length, we need to calculate the energy-weighted arc length $l_{i}$ based on the current point set $\left\{\boldsymbol{u}_{i}\right\}$ of the curve

$$
l_{i}=l_{i-1}+W\left(\frac{F\left(\boldsymbol{u}_{i-1}\right)+F\left(\boldsymbol{u}_{i}\right)}{2}\right)\left|\boldsymbol{u}_{i}-\boldsymbol{u}_{i-1}\right|, \quad i=1, \ldots, N,
$$

where $l_{0}=0$ and $W(x)$ is weight function which can be some well-selected positive and increasing functions for $x \in R$. The remaining processes are same as those in the first parametrization method.

In addition, the saddle point of the energy landscape can be located by the climbing string method [56], which is a two-step algorithm similar to the methodology in [28]. Unlike the string method, in the first step, the climbing string method first evolves the string through the following dynamics

$$
\left\{\begin{array}{l}
\left(\boldsymbol{u}_{i}\right)_{t}=-\nabla F\left(\boldsymbol{u}_{i}\right), \quad i=1, \ldots, N-1, \\
\left(\boldsymbol{u}_{N}\right)_{t}=-\nabla F\left(\boldsymbol{u}_{N}\right)+2\left(\nabla F\left(\boldsymbol{u}_{N}\right), \hat{\gamma}\right) \hat{\gamma} .
\end{array}\right.
$$

Then the same string reparametrization is applied as the normal string method. These two steps are repeated until the convergence is attained and the final state $\boldsymbol{u}_{N}$ locates a saddle point of the energy $F$. More details of the climbing string method can be found in [56]. 


\section{Numerical experiment}

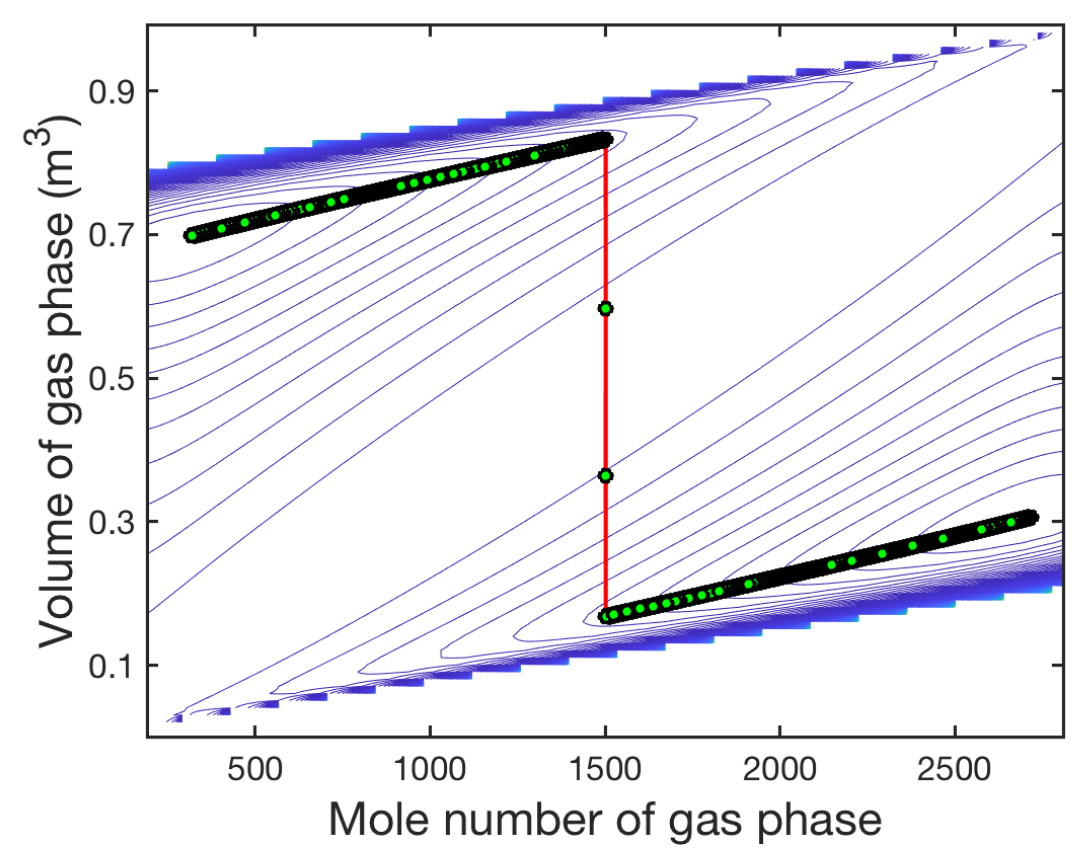

Figure 1: Minimum energy path of $\mathrm{nC}_{4}$

In this section, the MEPs of single-component and two-component VT flash models are calculated using the ETD-string scheme. Also, we compare the computed results given by the ETD-string scheme with the reference data for the single-component example, and with numerical results for the two-component example computed by the popular VT flash technique, to demonstrate the feasibility of the proposed scheme in dealing with VT flash problems. In the following examples, the total volume $V$ is assumed to be $1 \mathrm{~m}^{3}$. Table 1 presents compositional parameters of all the investigated species.

Table 1: Compositional parameters of all the investigated species in numerical examples

\begin{tabular}{ccccc}
\hline Component & $T_{c, i}(\mathrm{~K})$ & $P_{c, i}(\mathrm{MPa})$ & $M_{w, i}(\mathrm{~g} / \mathrm{mol})$ & $\omega_{i}$ \\
\hline $\mathrm{C}_{1}$ & 190.56 & 4.599 & 16.04 & 0.0110 \\
$\mathrm{nC}_{4}$ & 425.12 & 3.796 & 58.12 & 0.2010 \\
$\mathrm{nC}_{5}$ & 469.74 & 3.370 & 72.15 & 0.2510 \\
\hline
\end{tabular}




\subsection{Single-component case}

In this example, we calculate the MEP of the VT flash model for normal butane $\left(\mathrm{nC}_{4}\right)$ at temperature $T=350 \mathrm{~K}$. The total mole number is $3000 \mathrm{~mol}$ and the time step is $10^{-6}$. During the calculation process, the MEP was approximated using the string with 100 points and the initial string is the linear interpolation between $(316,0.69)$ and $(2700,0.3)$. For each point on the string, a gradient flow calculation is performed at each time step. The two end points of the string will eventually converge to the local minima. Cubic spline interpolation is used to redistribute the points of the string at each time step in terms of equal arc length. The whole process evolves until

$$
\max \frac{1}{\Delta t}\left|u_{i}^{n+1}-u_{i}^{n}\right|<\text { tol }=10^{-8} .
$$

Figure 1 shows the calculated MEP of $\mathrm{nC}_{4}$ at the given condition. Related saddle point $(1500,0.5)$ can be obtained by using the climbing string method.

Then the performance of the Rosenbrock-Euler-ETD scheme on the single-component VT flash problem is tested. To confirm the accuracy and credibility of the proposed algorithm, the computed results are compared with the reference data [55]. Numerical experiments are conducted in two ways: A) fixing the total mole number $N$ and changing the temperature $T$; $B$ ) fixing the temperature $T$ and changing the total mole number $N$.

Figure 2 presents the molar density of gaseous $\mathrm{nC}_{4}$ at the equilibrium state as a function of temperature at $N=3000 \mathrm{~mol}$. As temperature increases, the molar density of gaseous $\mathrm{nC}_{4}$ climbs up because an increasing number of molecules evaporate into the gas phase. The inset figures show the differences between the computed results of the proposed scheme and reference data, which cannot be clearly seen from the parent figure. Overall, the molar densities calculated by the Rosenbrock-Euler-ETD scheme agree with the reference data very well. Table 2 exhibits the equilibrium mole number, volume and molar density of gaseous $\mathrm{nC}_{4}$ change with the total mole number at fixed temperature. The constant molar densities can be explained in terms of the Gibbs phase rule. Since we fix the temperature, for a single-component system, the pressure is fixed as well. The densities of gas and liquid phase keep constant so that the variation of gas volume is identically proportional to the variation of the mole number of gas phase. It can be seen from Table 2 that the proposed scheme accurately calculates phase molar densities for the investigated cases. The convergence performance of the proposed method is verified in Table 3, from which it clearly shows that the ETD-string scheme is a first order scheme (we use the numerical solution under $d t=1^{-10}$ as the exact solution since our models do 
Table 3: Numerical order of Rosenbrock-Euler ETD-string scheme of single-component VT flash model

\begin{tabular}{ccc}
\hline$\Delta t$ & Error & order \\
\hline $1 \mathrm{e}-5$ & $5.44089 \mathrm{e}-06$ & - \\
$1 \mathrm{e}-6$ & $5.3919 \mathrm{e}-7$ & 1.00392 \\
$1 \mathrm{e}-7$ & $4.90176 \mathrm{e}-8$ & 1.04139 \\
\hline
\end{tabular}

not have exact solution).

Table 2: Equilibrium mole number $\left(N_{g}\right)$, volume $\left(V_{g}\right)$ and molar density $\left(n_{g}\right)$ of gaseous $\mathrm{nC}_{4}$ at $T=350 \mathrm{~K}$ with total mole number $N=2000,3500,5000,6500,8000 \mathrm{~mol}$

\begin{tabular}{cccccc}
\hline$N$ & $N_{g}($ ETD $)$ & $V_{g}($ ETD $)$ & $n_{g}($ ETD $)$ & $n_{g}([19])$ & laboratory data \\
\hline 2000 & 326.3 & 0.812 & 402.2 & 402.1 & 402.4 \\
3500 & 254.9 & 0.634 & 402.2 & 402.1 & 402.4 \\
5000 & 184.2 & 0.458 & 402.2 & 402.1 & 402.4 \\
6500 & 113.0 & 0.281 & 402.2 & 402.1 & 402.4 \\
8000 & 41.8 & 0.104 & 402.2 & 402.1 & 402.4 \\
\hline
\end{tabular}

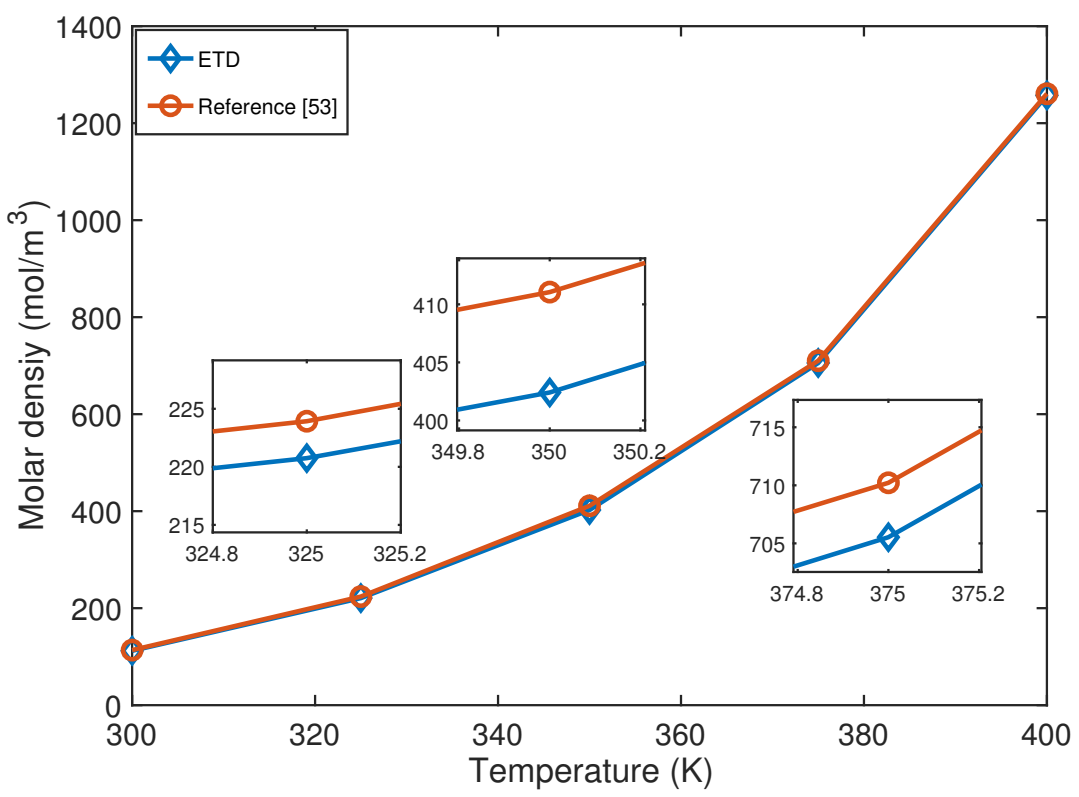

Figure 2: Equilibrium molar density of gaseous $\mathrm{nC}_{4}$ at $\mathrm{T}=300,325,350,375,400 \mathrm{~K}$ and the comparison with the reference data 
Table 4: Equilibrium mole number of gaseous $\mathrm{C}_{1}(1), \mathrm{nC}_{5}(2)$ and gas phase volume $\left(V_{g}\right)$ at $N=6000$ mol with temperature $T=300,325,350,375,400 \mathrm{~K}$

\begin{tabular}{|c|c|c|c|c|c|c|}
\hline$T$ & $N_{1 g}$ (ETD) & $N_{2 g}$ (ETD) & $V_{g}$ (ETD) & $N_{1 g}$ (VT flash) & $N_{2 g}$ (VT flash) & $V_{g}$ (VT flash) \\
\hline 300 & 2086.79 & 74.97 & 0.634 & 2086.85 & 75.00 & 0.633 \\
\hline 325 & 2062.08 & 145.12 & 0.615 & 2061.81 & 145.03 & 0.617 \\
\hline 350 & 2006.08 & 258.64 & 0.596 & 2006.07 & 258.75 & 0.596 \\
\hline 375 & 1911.12 & 444.89 & 0.568 & 1911.58 & 444.49 & 0.568 \\
\hline 400 & 1716.13 & 787.53 & 0.514 & 1716.50 & 787.51 & 0.514 \\
\hline
\end{tabular}

\subsection{Two-component case}

In practice, the pure-component fluid is very rare especially when investigating hydrocarbon fluids in the subsurface reservoirs. Thus, it is important that a numerical scheme enables to handle multicomponent mixtures. In this example, we consider a binary mixture of methane $\left(\mathrm{C}_{1}\right)$ and normal pentane $\left(\mathrm{nC}_{5}\right)$, and calculate the MEP of this binary system at $T=300 \mathrm{~K}$ using the same method for the single-component case. The total mole number $N$ is $6000 \mathrm{~mol}$, and the feed composition of $\mathrm{C}_{1}$ and $\mathrm{nC}_{5}$ is $z_{\mathrm{C}_{1}}=0.547413$ and $z_{\mathrm{nC}}=0.452587$. The binary interaction coefficient between $\mathrm{C}_{1}$ and $\mathrm{nC}_{5}$ is $k_{\mathrm{C}_{1}-\mathrm{nC}_{5}}=0.041$, and the time step is $10^{-4}$.

In comparison to the single-component model, the multicomponent model is more difficult to solve, and the main difficulty lies in the strong stiffness of the Hessian matrix. According to our numerical tests, this stiffness results from the volume change. In order to reduce the influence of volume change, we do variable substitution and replace $V_{1}$ by $\hat{V}_{1}$, where $V_{1}=\beta \times \hat{V}_{1}$. Here $\beta$ is a constant and we set $\beta=10^{-6}$ in this numerical experiment. Now the primary unknowns are $x=\left[N_{1,1}, \ldots, N_{M, 1}, \hat{V}_{1}\right]$, where $N_{i, 1}$ is the mole number of $i$ th component in phase 1 . In this example, the MEP was approximated using the string with 50 points and the initial string is a linear interpolation between $(2000,80,0.63)$ and $(1300,2600,0.37)$. Figure 3 shows the evolution of the string from the initial state to the stable state by every 2000 steps. Figure 4 shows the total energy of each point at the stable state. We can clearly see from Figure 4 that along the calculated MEP the total energy of the system overcomes the energy barrier which separates two minimum points of original system.

Table 4 presents the equilibrium mole number of gaseous $\mathrm{C}_{1}$ and $\mathrm{nC}_{5}$ and gas phase volume at $N=6000$ mole with five different temperatures. It can be seen that the mole numbers calculated by the Rosenbrock-Euler-ETD scheme shows high agreement with the numerical results computed by the conventional VT flash method [16]. This demonstrates that our new algorithm is also feasible for complex multicomponent systems. 


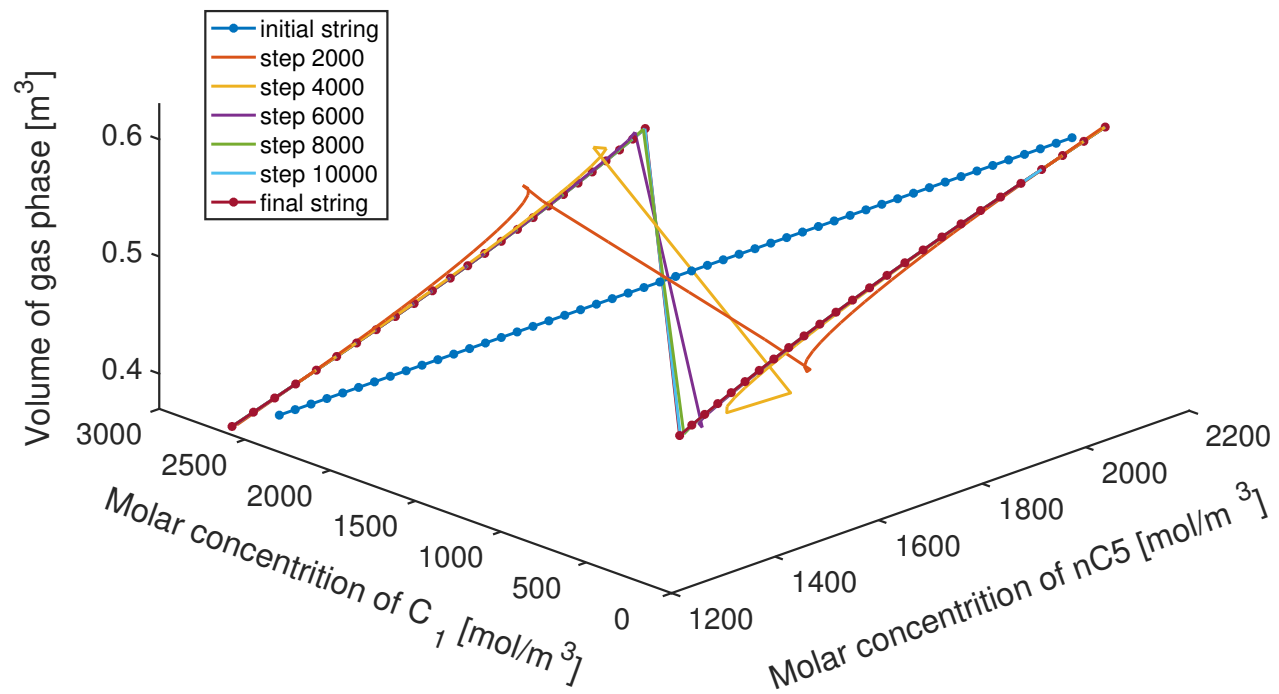

Figure 3: The evolution of string from the initial state to the final state for the binary mixture of $\mathrm{C}_{1}$ and $\mathrm{nC}_{5}$

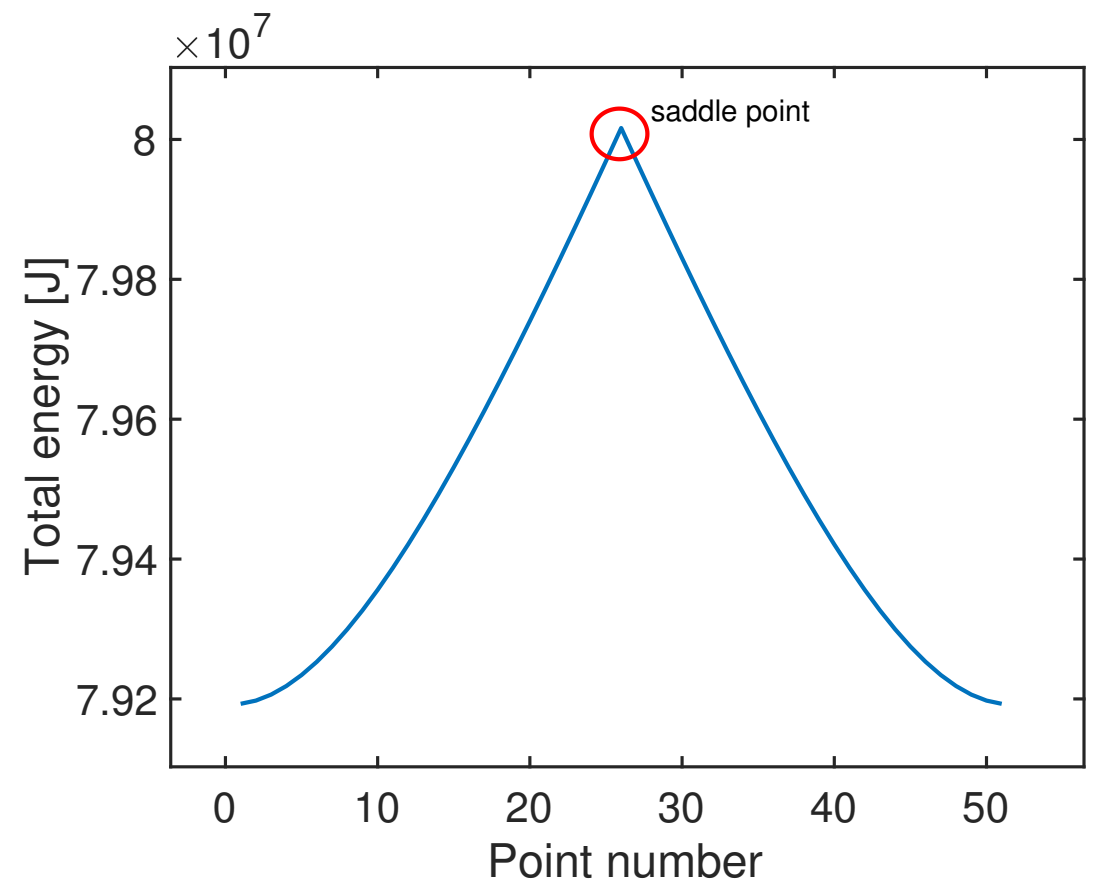

Figure 4: Total energy of each point at the stable state for the binary mixture of $\mathrm{C}_{1}$ and $\mathrm{nC}_{5}$ 


\section{Conclusion}

In this study, the ETD-String method is employed to efficiently compute the MEPs of single-component and two-component VT flash models with the Peng-Robinson equation of state. In order to avoid the computational difficulty caused by the strong stiffness, the Rosenbrock-type ETD scheme is used to evolve points of the string at each time step. The local minima have been successfully connected by a MEP of the given model and its saddle point is accurately determined. The equilibrium solutions computed by the Rosenbrock-ETD scheme show good agreement with the published data for the singlecomponent example and with numerical results using the popular VT flash methods for the two-component example. This work has discussed the relation between the local minima of a single-component and two-component VT flash models and their saddle points for the first time. However, it is still a nontrivial task to obtain the high-order saddle points and to find the global minimum energy point for a multicomponent VT flash system. This is the subject of our future work and will help us grasp the global energy landscape of VT flash problems.

\section{Acknowledgments}

The authors greatly thank for the support from the National Natural Science Foundation of China (grant number 51874262, 51936001,11802090, 12050002) and the Research Funding from King Abdullah University of Science and Technology (KAUST) through the grants BAS/1/1351-01, URF/1/4074-01, URF/1/3769-01, and REP/1/2879-01.

\section{A Parameters of the Peng-Robinson equation of state}

The Peng-Robinson equation of state has the following form

$$
P(N, V, T)=\frac{N R T}{V-B}-\frac{A}{V^{2}+2 B V-B^{2}},
$$

where $A=a N^{2}$ and $B=b N . N=\sum_{i} N_{i}$ is the total mole number. The energy parameter $a$ and co-volume parameter $b$ of a fluid mixture can be computed based on the classical Van der Waals mixing rule such that

$$
a=\sum_{i} \sum_{j} x_{i} x_{j}\left(a_{i} a_{j}\right)^{1 / 2}\left(1-k_{i j}\right), \quad b=\sum_{i} x_{i} b_{i}
$$


In the above expressions, $k_{i j}$ is the binary interaction coefficient, $a_{i}$ and $b_{i}$ is the energy parameter and co-volume parameter of pure component

$$
\begin{gathered}
a_{i}=0.45724 \frac{R^{2} T_{c, i}^{2}}{P_{c, i}}\left(1+m_{i}\left(1-\sqrt{\frac{T}{T_{c, i}}}\right)\right)^{2}, \\
b_{i}=0.07780 \frac{R T_{c, i}}{P_{c, i}}
\end{gathered}
$$

where $P_{c, i}$ and $T_{c, i}$ denote the critical pressure and critical temperature of $i$ th component. The coefficient $m_{i}$ is a function of the acentric factor $\omega_{i}$ of pure substance

$$
m=\left\{\begin{array}{l}
0.37464+1.54226 \omega_{i}-0.26992 \omega_{i}^{2}, \quad \omega_{i} \leq 0.49 \\
0.379642+1.485030 \omega_{i}-0.164423 \omega_{i}^{2}+0.016666 \omega_{i}^{3}, \quad \omega_{i}>0.49
\end{array}\right.
$$

\section{References}

[1] Chen, Z. (2007). Reservoir simulation: mathematical techniques in oil recovery. SIAM.

[2] Poormohammadian, S.J., Lashanizadegan, A., Salooki, M.K. (2015). Modelling VLE data of $\mathrm{CO}_{2}$ and $\mathrm{H}_{2} \mathrm{~S}$ in aqueous solutions of $\mathrm{N}$-methyldiethanolamine based on non-random mixing rules. International Journal of Greenhouse Gas Control, 42, 87-97.

[3] Wang, T., El Ahmar, E., Coquelet, C. (2017). Alkane solubilities in aqueous alkanolamine solutions with CPA EoS. Fluid Phase Equilibria, 434, 93-101.

[4] Wang, T., El Ahmar, E., Coquelet, C., Kontogeorgis, G. M. (2018). Improvement of the PRCPA equation of state for modelling of acid gases solubilities in aqueous alkanolamine solutions. Fluid Phase Equilibria, 471, 74-87.

[5] Anderson, F.E., Prausnitz, J.M. (1986). Inhibition of gas hydrates by methanol. AIChE journal, 32(8), 1321-1333.

[6] Pedersen, K.S., Michelsen, M.L., Fredheim, A.O. (1996). Phase equilibrium calculations for unprocessed well streams containing hydrate inhibitors. Fluid Phase Equilibria, 126(1), 1328.

[7] Haghighi, H., Chapoy, A., Burgess, R., Tohidi, B. (2009). Experimental and thermodynamic modelling of systems containing water and ethylene glycol: Application to flow assurance and gas processing. Fluid Phase Equilibria, 276(1), 24-30.

[8] Burgass, R., Chapoy, A., Li, X. (2018). Gas hydrate equilibria in the presence of monoethylene glycol, sodium chloride and sodium bromide at pressures up to $150 \mathrm{MPa}$. The Journal of Chemical Thermodynamics, 118, 193-197.

[9] Sabbagh, O., Akbarzadeh, K., Badamchi-Zadeh, A., Svrcek, W.Y., Yarranton, H.W. (2006). Applying the PR-EoS to asphaltene precipitation from n-alkane diluted heavy oils and bitumens. Energy \& fuels, 20(2), 625-634.

[10] Shirani, B., Nikazar, M., Mousavi-Dehghani, S.A. (2012). Prediction of asphaltene phase behavior in live oil with CPA equation of state. Fuel, 97, 89-96.

[11] Jindrová, T., Mikyška, J., Firoozabadi, A. (2016). Phase Behavior Modeling of Bitumen and Light Normal Alkanes and CO2 by PR-EOS and CPA-EOS. Energy \& Fuels, 30(1), 515-525.

[12] Michelsen, M.L. (1982). The isothermal flash problem. Part I. Stability. Fluid phase equilibria, 9(1), 1-19. 
[13] Michelsen, M.L. (1982). The isothermal flash problem. Part II. Phase-split calculation. Fluid phase equilibria, 9(1), 21-40.

[14] Soave, G. (1972). Equilibrium constants from a modified Redlich-Kwong equation of state. Chemical engineering science, 27(6), 1197-1203.

[15] Peng, D., Robinson, D.B. (1976). A new two-constant equation of state. Industrial and Engineering Chemistry Fundamentals, 15(1), 59-64.

[16] Jindrová, T., Mikyška, J. (2013). Fast and robust algorithm for calculation of two-phase equilibria at given volume, temperature, and moles. Fluid Phase Equilibria, 353, 101-114.

[17] Li, Y., Kou, J., Sun, S. (2018). Thermodynamically stable two-phase equilibrium calculation of hydrocarbon mixtures with capillary pressure. Industrial \& Engineering Chemistry Research, 57(50), 17276-17288.

[18] Sun, S. (2019). Darcy-scale phase equilibrium modeling with gravity and capillarity. Journal of Computational Physics, 399, 108908.

[19] Nichita, D.V. (2019). Density-based phase envelope construction including capillary pressure. Fluid Phase Equilibria, 498, 33-44.

[20] Travalloni, L., Castier, M., Tavares, F.W. (2014). Phase equilibrium of fluids confined in porous media from an extended Peng-Robinson equation of state. Fluid Phase Equilibria, $362,335-341$.

[21] Luo, S., Lutkenhaus, J.L., Nasrabadi, H. (2018). Multiscale fluid-phase-behavior simulation in shale reservoirs using a pore-size-dependent equation of state. SPE Reservoir Evaluation \& Engineering, 21(04), 806-820.

[22] Li, Y., Qiao, Z., Sun, S., Zhang, T. (2020). Thermodynamic modeling of $\mathrm{CO}_{2}$ solubility in saline water using NVT flash with the cubic-plus-association equation of state. Fluid Phase Equilibria, 112657.

[23] Polívka, O., Mikyška, J. (2014). Compositional modeling in porous media using constant volume flash and flux computation without the need for phase identification. Journal of Computational Physics, 272, 149-169.

[24] Pan, H., Firoozabadi, A. (1998). Complex multiphase equilibrium calculations by direct minimization of Gibbs free energy by use of simulated annealing. SPE Reservoir Evaluation \& Engineering, 1(01), 36-42.

[25] Zhu, Y., Wen, H., Xu, Z. (2000). Global stability analysis and phase equilibrium calculations at high pressures using the enhanced simulated annealing algorithm. Chemical Engineering Science, 55(17), 3451-3459.

[26] Nichita, D.V., Gomez, S., Luna, E. (2002). Multiphase equilibria calculation by direct minimization of Gibbs free energy with a global optimization method. Computers \& chemical engineering, 26(12), 1703-1724.

[27] Zhang, H., Bonilla-Petriciolet, A., P Rangaiah, G. (2011). A review on global optimization methods for phase equilibrium modeling and calculations. The Open Thermodynamics Journal, 5(1).

[28] Weinan E, Ren W, Vanden-Eijnden E. (2008). Simplified and improved string method for computing the minimum energy paths in barrier-crossing events. Journal of Chemical Physics, 126(16), 164103.

[29] Yin, J., Yu, B., Zhang, L. (2020). Searching the solution landscape by generalized high-index saddle dynamics, arXiv preprint arXiv:2002.10690.

[30] Yin J., Zhang L., Zhang P. (2019). High-Index Optimization-Based Shrinking Dimer Method for Finding High-Index Saddle Points. SIAM Journal on Scientific Computing, 41(6), A3576A3595. 
[31] Yin, J., Wang, Y., Chen, J.Z., Zhang, P., Zhang, L. (2020). Construction of a pathway map on a complicated energy landscape. Physical Review Letters, 124(9), 090601.

[32] Ulitsky, A., Elber, R. (1990). A new technique to calculate steepest descent paths in flexible polyatomic systems. The Journal of chemical physics, 92(2), 1510-1511.

[33] Fischer, S., Karplus, M. (1992). Conjugate peak refinement: an algorithm for finding reaction paths and accurate transition states in systems with many degrees of freedom. Chemical physics letters, 194(3), 252-261.

[34] Henkelman, G., Uberuaga, B. P., Jnsson H. (2000). A climbing image nudged elastic band method for finding saddle points and minimum energy paths. The Journal of Chemical Physics, 113(22), 9901-9904.

[35] Weinan E., Ren W., Vanden-Eijnden E. (2002). String method for the study of rare events. Physical Review B, 66(5), 052301.

[36] Berne B.J., Ciccotti G., Coker D.F. (1998). Classical and quantum dynamics in condensed phase simulations: Proceedings of the International School of Physics. World Scientific, Singapore.

[37] Taflove, A., Hagness, S.C. (2005). Computational electrodynamics: the finite-difference timedomain method. Artech house, London.

[38] Beylkin, G., Keiser, J.M., Vozovoi, L. (1998). A new class of time discretization schemes for the solution of nonlinear PDEs. Journal of Computational Physics, 147(2), 362-387.

[39] Cox, S.M., Matthews, P.C. (2002). Exponential time differencing for stiff systems. Journal of Computational Physics, 176(2), 430-455.

[40] Du, Q., Zhu, W. X. (2004). Stability analysis and application of the exponential time differencing schemes. Journal of Computational Mathematics, 22, 200-209.

[41] Du, Q., Zhu, W. (2005). Analysis and applications of the exponential time differencing schemes and their contour integration modifications. BIT Numerical Mathematics, 45(2), 307-328.

[42] Du Q., Ju L., Li X., Qiao Z. Maximum bound principles for a class of semilinear parabolic equations and exponential time differencing schemes[J]. arXiv preprint arXiv:2005.11465, 2020.

[43] Ju L., Li X., Qiao Z., Yang J. Maximum bound principle preserving integrating factor RungeKutta methods for semilinear parabolic equations[J]. arXiv preprint arXiv:2010.12165, 2020.

[44] Du Q., Ju L., Li X., Qiao Z., Maximum bound principles for a class of semilinear parabolic equations and exponential time-differencing schemes, SIAM Rev. 63 (2021) 317-359.

[45] Hochbruck, M., Ostermann, A., Schweitzer, J. (2006). Exponential integrators of Rosenbrocktype. Oberwolfach Reports, 3, 1107-1110.

[46] Hochbruck, M., Ostermann, A., Schweitzer, J. (2009). Exponential Rosenbrock-type methods. SIAM Journal on Numerical Analysis, 47(1), 786-803.

[47] Firoozabadi, A. (2015). Thermodynamics and applications of hydrocarbon energy production. McGraw Hill Professional.

[48] Mikyška, J., Firoozabadi, A. (2012). Investigation of mixture stability at given volume, temperature, and number of moles. Fluid Phase Equilibria, 321, 1-9.

[49] Jindrová, T., Mikyška, J. (2015). General algorithm for multiphase equilibria calculation at given volume, temperature, and moles. Fluid Phase Equilibria, 393, 7-25.

[50] Nichita, D.V. (2017). Fast and robust phase stability testing at isothermal-isochoric conditions. Fluid Phase Equilibria, 447, 107-124.

[51] Nichita, D.V. (2018). New unconstrained minimization methods for robust flash calculations at temperature, volume and moles specifications. Fluid Phase Equilibria, 466, 31-47. 
[52] Gill, P.E., Murray, W. (1974). Newton-type methods for unconstrained and linearly constrained optimization. Mathematical Programming, 7(1), 311-350.

[53] Schnabel, R.B., Eskow, E. (1999). A revised modified Cholesky factorization algorithm. SIAM Journal on optimization, 9(4), 1135-1148.

[54] Higham, N.J., Functions of Matrices: Theory and Computation, SIAM, Philadelphia, PA, 2008.

[55] Younglove, B.A., Ely, J.F. (1987). Thermophysical properties of fluids. II. Methane, ethane, propane, isobutane, and normal butane. Journal of Physical and Chemical Reference Data, 16(4), 57-798.

[56] Ren, W., Vanden-Eijnden, E. (2013). A climbing string method for saddle point search. The Journal of chemical physics, 138(13), 134105. 